\title{
A tomographic technique for the simultaneous imaging of temperature, chemical species, and pressure in reactive flows using absorption spectroscopy with frequency-agile lasers
}

\author{
Weiwei Cai (蔡伟伟) and Clemens F. Kaminski ${ }^{\text {a) }}$ \\ Department of Chemical Engineering and Biotechnology, University of Cambridge, Cambridge CB2 3RA, UK
}

(Received Month X, XXXX; revised Month X, XXXX; accepted Month X, XXXX; posted Month X)

\begin{abstract}
This paper proposes a technique that can simultaneously retrieve distributions of temperature, concentration of chemical species, and pressure based on broad bandwidth, frequency-agile tomographic (FAT) absorption spectroscopy. The technique holds particular promise for the study of dynamic combusting flows. A proof-of-concept numerical demonstration is presented using representative phantoms to model conditions typically prevailing in near-atmospheric or high pressure flames. The simulations reveal both the feasibility of the proposed technique and its robustness. Our calculations indicate precisions of $\sim 70 \mathrm{~K}$ at flame temperatures and $\sim 0.05$ bar at high pressure from reconstructions featuring as much as $5 \%$ Gaussian noise in the projections.
\end{abstract}

(C) 2014 AIP Publishing LLC.

\begin{abstract}
Absorption spectroscopy has become an indispensable tool for flow diagnostics due to its species-specificity, simplicity of implementation, and versatility in measuring multiple flow parameters such as temperature, concentration of target species, and pressure ${ }^{1}$. However, traditional absorption spectroscopy is a line-of-sight technique, which reflects only averaged properties along the laser path without providing any spatially-resolved information. Over the past decade, much effort has been devoted to the combination of tomographic methods, originating from the medical field, with those based on gas phase absorption spectroscopy. It has for example become possible to resolve features in nonuniform flow fields from one, or at most two, distinct absorption features ${ }^{2-7}$. To permit successful tomographic reconstructions, line-of-sight measurements must be performed along multiple directions using either parallel or fanned beam arrangements, both require mechanical means for angular beam displacement and thus spatial resolution is obtained at the cost of reduced temporal resolution. This is a problem for application in fast-changing turbulent flames. Recently a technique called multispectral tomography has been developed ${ }^{8-11}$ which requires no rotational parts and is enabled by the advent of so-called frequency-agile laser sources. Here temporal resolution only depends on laser repetition- and detector-sampling rates. By utilizing highrepetition rate pulsed supercontinuum radiation ${ }^{12,13}$, it is furthermore possible to perform spatially-resolved flame diagnostics on multiple species and other flame parameters, at ultra-high repetition rates (on the order of $\mathrm{MHz}$ ).

Unfortunately, all previous techniques rely on the assumption that the pressure is constant, uniform and known. In some environments this invalidates their application; e.g. in the supersonic flow field within a scramjet the prevailing
\end{abstract}

shock waves lead to dramatic pressure drops due to nonuniform and localized heat release. However, to establish pressure distributions is highly desirable since these relate directly to thrust and influence the highest Mach numbers achievable. The aim of the present work is to defeat aforementioned limitations and to provide the conceptual framework for a novel tomographic technique that measures not only spatially resolved temperature and species concentration fields but also the pressure distribution without sacrificing temporal resolution. We term the technique frequency-agile tomography (FAT).

To monitor multiple chemical species simultaneously in the reactive flow, a broad bandwidth supercontinuum laser source can be used to cover a large number spectral signatures $^{12}$. The supercontinuum can be generated by pumping short (ps or less) pulses into a photonic crystal fiber ${ }^{14}$ where nonlinear effects such as self-phase modulation, stimulated Raman scattering, and four-wave mixing lead to massive spectral broadening ${ }^{15}$. The color spectrum of the ensuing broad bandwidth pulses can then be temporally dispersed via passage through a long dispersive fiber. A spectrum can thus be recorded for each pulse simply by recording the time resolved signal on a high bandwidth photo-detector and sweep rates of up to $1 \mathrm{~nm}$ per ns are possible $^{13}$. Detailed implementation of this ultra-fast wavelength-sweeping technique can be found in References ${ }^{16-18}$. For the tomographic technique presented here the supercontinuum is split into multiple beams to map out the ROI. This is shown in the Panel (a) of Fig. 1 which also serves to illustrate the mathematical formulation of the problem. The region of interest (ROI) is discretized into a grid of $M \times N$ squares along the $x$ and $y$ directions respectively. One of the laser beams positioned at the $j$ th location, denoted

\footnotetext{
a) Electronic email: cfk23@cam.ac.uk
} 
by $\ell_{j}$, is directed along the line-of-sight (LOS) across the non-uniform combustion field (left sub-panel). Since the absorbance is accumulative over the absorption path length, the overall absorption spectrum along the LOS is obtained by summing up the spectra of each resolution element traversed by the laser beam (indicated by blue squares in Fig 1a). This leads to a projection spectrum (right sub-panel) $p(\ell, \lambda)$ with $\lambda$ indicating the swept wavelength. The absorption peaks that can be used for tomographic inversion are named as $\lambda_{i}$ with the subscript specifying wavelength indices. The absorbance by the targeting species at $\lambda_{i}$ is the summation of contributions from neighboring transitions, as expressed by the following equation

$$
\begin{aligned}
& p\left(\ell_{j}, \lambda_{i}\right)=\sum_{q} \alpha_{q}^{j, i}\left(T_{q}, X_{q}, P_{q}\right) \\
& =\sum_{q} \sum_{k} S\left(\lambda_{k}, T_{q}\right) \cdot X_{q} \cdot \phi\left(\lambda_{k}-\lambda_{i}, T_{q}, X_{q}, P_{q}\right) \cdot P_{q} \cdot \Delta
\end{aligned}
$$

where $q$ is the index running through all grid squares crossed by the $j$ th laser beam; $\lambda_{k}$ the center wavelength of the $k$ th contributing transition $\alpha^{j, i}$ the absorbance at the $i$ th peak wavelength in the $q$ th square; $T_{q}, X_{q}$, and $P_{q}$ the corresponding temperature, concentration, and total pressure, respectively; $S$ is the line strength of the contributing transition; $\Phi$ the normalized Voigt lineshape function; and $\Delta$ is the grid spacing. Panel (b) and (c) of Fig. 1. illustrate how different flow parameters affect the spectrum of a specific absorption feature. Panel (b) contains four example spectra modeled using data from a spectroscopic database ${ }^{19,20}$ with Panel (c) showing the corresponding normalized spectra for better comparison. Each spectrum is modeled for a different combination of $T_{q}, X_{q}$, and $P_{q}$. Water vapor was chosen in the model due to its prevalence and abundance in hydrocarbon/hydrogen flames, and the availability of frequency agile laser sources to target this species. Collisional and Doppler are the dominant homogeneous and inhomogeneous broadening mechanisms ${ }^{21}$ and overall broadening is a convolution of the two, well approximated by a Voigt profile ${ }^{22}$. At atmospheric pressure, the typical full width half maximum (FWHM) of absorption features is $\sim 0.1$ $\mathrm{cm}^{-1}$ or $\sim 0.02 \mathrm{~nm}$ in the near infrared and this is easily resolved by frequency-agile sensing as we have previously shown ${ }^{12,16,17}$. Also, the features broaden quickly as pressure increases which makes them easier to resolve. It is found that, for the particular feature shown, absorption intensity decreases with temperature and that the FWHM changes slightly due to the impact of temperature on both line strength and shape. Other spectral features may have different dependencies on temperature. We find furthermore that absorption strength varies almost linearly with concentration. Finally, the pressure not only acts as a linear scaling factor for absorbance, it also broadens and flattens the line shape. The line center exhibits small red shifts caused by pressure. However, the problem is further complicated by the non-uniform distributions of flow parameters, which make it impossible to quantify and interpret traditional line-of-sight measurements. Looking at this problem from another perspective, all information about the flow parameters is contained in $p\left(\ell_{i}, \lambda_{i}\right)$; and through suitable mathematical formulation and post-processing, the entanglement of all these factors can be resolved and the spatially-resolved flow field information can be recovered by the so-called FAT algorithm.
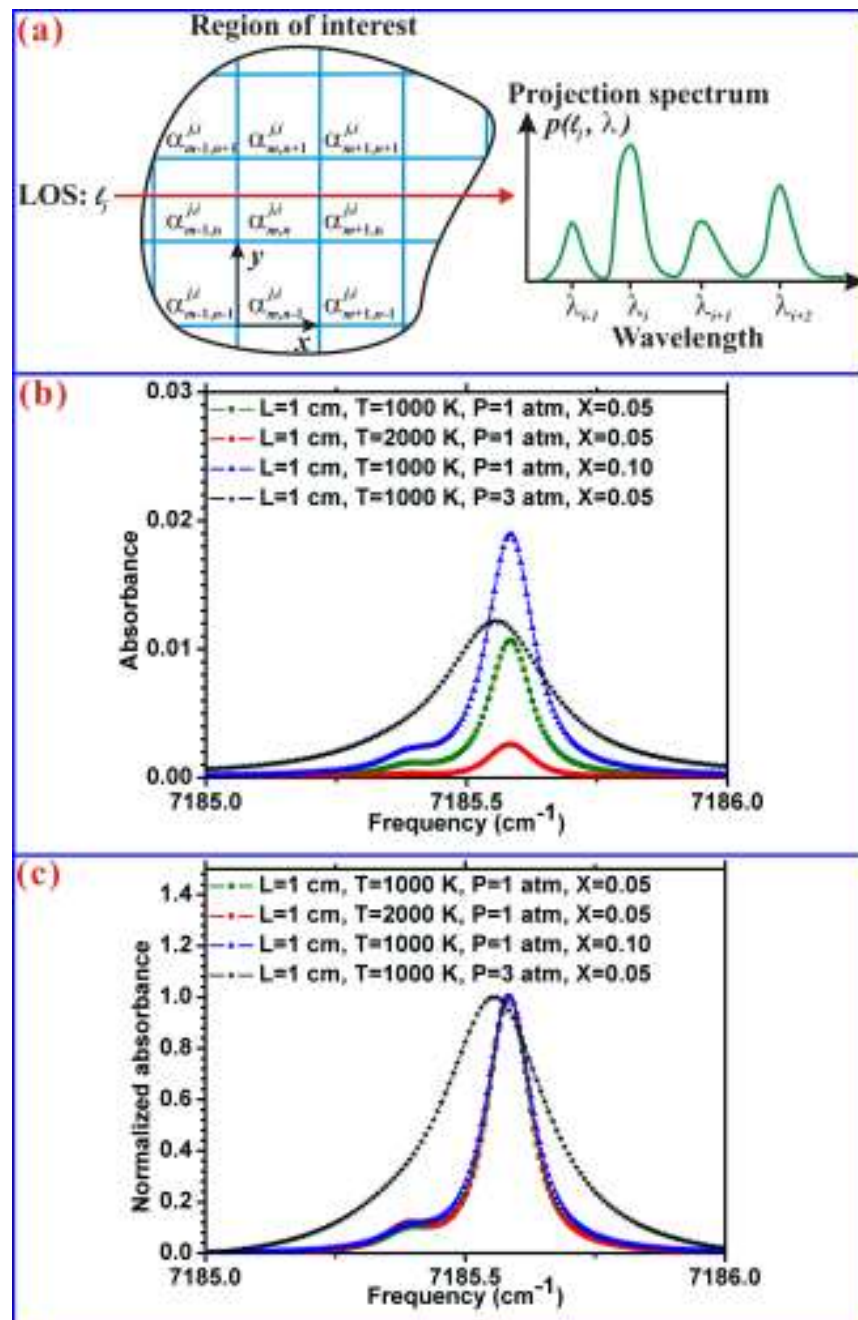

Fig. 1. (Color online) Mathematical formulation of the multispectral tomographic problem.

The FAT algorithm seeks to reconstruct distributions over ROI with a limited number of projections and can be formulated into an algebraic nonlinear minimization problem with a cost function defined as

$$
D=\sum_{j=1}^{j} \sum_{i=1}^{\prime}\left[1-p_{c}\left(\ell, \lambda_{i}, T_{q}, X_{q}, P_{q}\right) / p_{m}\left(\ell, \lambda_{j}\right)\right]^{2}
$$

where $J$ and $I$ denote the total number of laser beams and peak wavelengths used, respectively; $p_{m}\left(\ell_{i}, \lambda_{i}\right)$ the measured projection at the wavelength $\lambda_{i}$ along along the $j$ th laser beam; and $p_{c}\left(\ell_{i}, \lambda_{i}\right)$ the corresponding projection computed with reference to an appropriate spectroscopic database. The cost function, $D$, provides a quantitative measure of the similarity between the reconstructed and the actual profiles. The terms contributing to $D$ are the errors in projections, normalized to ensure their equal weighting in the optimization. In an ideal case, where measurements are noise-free and the spectroscopic database is accurate, $D$ reaches its global minimum (zero) when the reconstructions match the true profiles. As for other typical minimization problems additional information, such as smoothness 
conditions due to thermal and mass diffusion, can be easily incorporated into the inversion process by adding regularization terms into the cost function. For example the smoothness of temperature can be implemented with the regularization term:

$$
R_{T}\left(\vec{T}^{r e c}\right)=\sum_{m=1}^{M} \sum_{n=1}^{N}\left[\left(\sum_{i=m-1}^{m+1} \sum_{j=n-1}^{n+1} T_{i, j}^{r e c}-T_{m, n}^{r e c}\right) / 8\right]
$$

Obviously, $R_{T}$ decreases as the distribution becomes smoother and reaches zero when the temperature remains constant (flat profile) across the ROI. Similar terms $R_{X}$ and $R_{P}$ can be implemented analogously for concentration and pressure, respectively. The cost function can thus be modified as

$$
F=D+\gamma_{T} \cdot R_{T}\left(T^{r c c}\right)+\gamma_{x} \cdot R_{X}\left(X^{r e c}\right)+\gamma_{P} \cdot R_{P}\left(P^{r e c}\right)
$$

where $\gamma_{T}, \gamma_{X}$, and $\gamma_{P}$ are weighting parameters employed to regulate the relative significance of a priori (smoothness) and posteriori (measured projections) information. The minimization problem can then be solved via use of a global minimizer. Here, we adopt the well-established Simulated Annealing (SA) algorithm to obtain distributions. More details on the use of regularization, determination of optimal regularization parameters, and the SA algorithm can be found in $^{8}$.

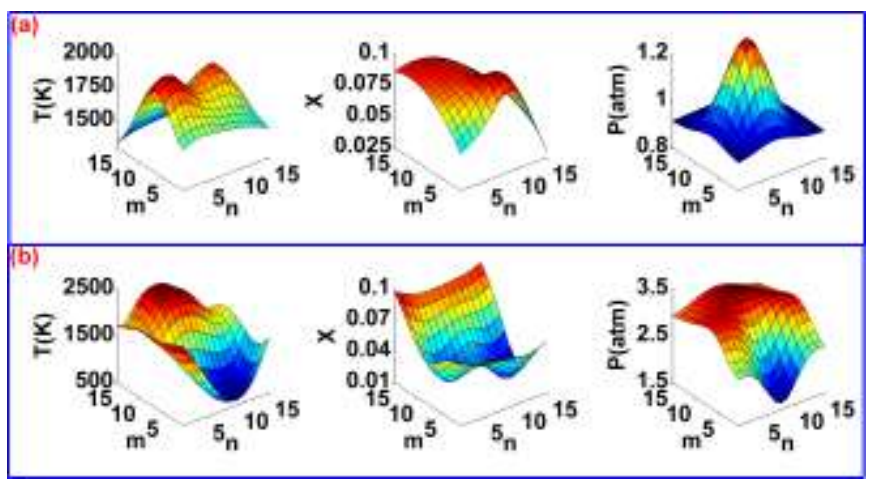

Fig. 2. (Color online) Temperature, species concentration, and pressure Phantoms generated for use in numerical simulations.

To provide a proof-of-concept demonstration and validation of our method, we constructed two sets of artificial multi-modal phantoms to mimic conditions encountered in real world reacting flows. These are depicted in Panel (a) and (b) of Fig. 2 respectively. The second set was generated according to the numerical study of supersonic combustion detailed $\mathrm{in}^{23}$. The ROI was meshed using a square grid containing $15 \times 15$ cells. For each cell we tested the ability of the algorithm to recover three unknowns, i.e. $T, X$, and $P$ respectively, resulting in 675 variables in total. To probe all the cells in the ROI, a simple probing configuration was assumed, containing of 15 horizontal ( $x$ direction) and 15 vertical ( $y$ direction) laser beams. Artificial random noise was imposed on "accurate" projections, which were calculated using data from a spectroscopic database ${ }^{19,20}$ to approximate a practical and noisy measurement situation. To quantify the reconstruction fidelity for temperature, the relative error was defined as the normalized overall deviation of the temperature reconstruction from the phantom. This is mathematically expressed as

$$
e_{T}=\left\|\vec{T}^{\text {rec }}-\vec{T}^{\text {true }}\right\|_{1} /\left\|\vec{T}^{\text {true }}\right\|_{1}
$$

where $\left\|\bar{T}^{\text {true }}\right\|$ is the true temperature distribution in a vector form and \|\|$_{1}$ denotes the Manhattan norm of a vector. Similar definitions can be analogously defined for concentration and pressure distributions.

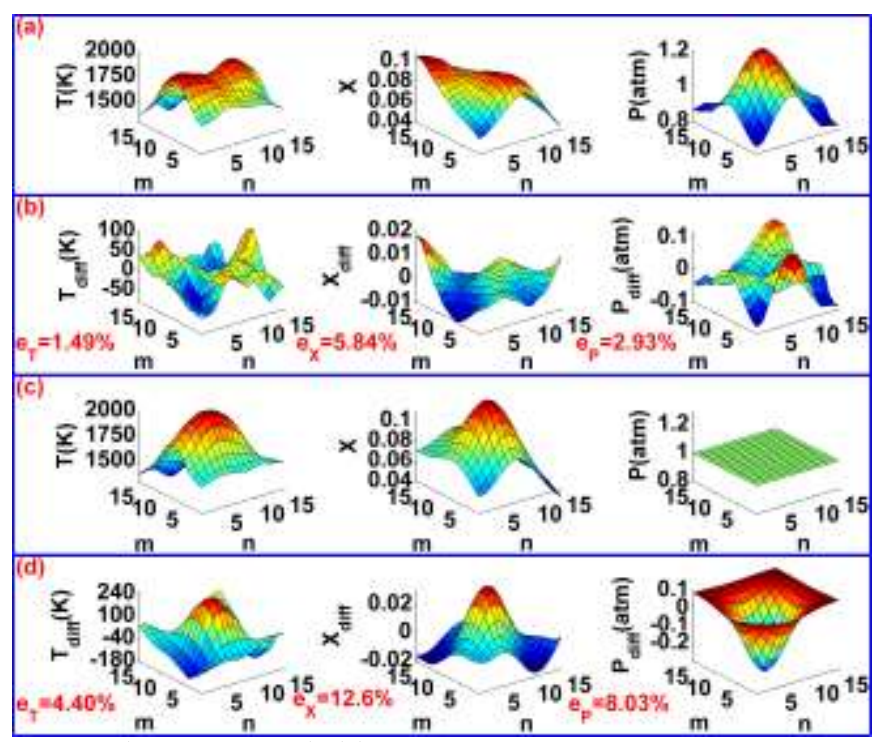

Fig. 3. (Color online) (a) Reconstructed distributions with 5\% Gaussian noise added to the projections; (b) Error contours for Panel (a); (c) Counterpart of Panel (a) assuming constant pressure distribution; and (d) Error Contours for Panel (c).

Panel (a) of Fig. 3 contains example reconstructions for the first phantom set from a simulation case where 5\% Gaussian noise was added to the projections and 23 peak wavelengths (690 projections) were used in the inversion process. Panel (b) presents error contours accordingly. As can be seen, the maximum error happens on the corners and edges where the smoothness conditions are more weakly enforced relative to more central domains of the ROI. The average error for temperature $\left(e_{T}\right)$ is $1.5 \%$ which corresponds an absolute value of $\sim 30 \mathrm{~K}$ at the temperatures considered. Panel (c) shows reconstructions using the same noisy projections but here the pressure is assumed to be constant, a commonly adopted assumption in the literature. Clearly, now the spatial distribution of the temperature field is dramatically affected and bears no resemblance to the true field. Panel (d) depictes the resulting error distributions. Relative errors are between 2-4 times larger than using a FAT algorithm that also recovers pressure fields (b).

The simulation results for the second phantom set are contained in Fig. 4 clearly manifesting the outperformance of the new technique over current methods. The reconstructions successfully capture multimodal features with average errors of only $\sim 70 \mathrm{~K}$ in temperature and 0.05 bar in pressure. It is worth noticing that these precisions are estimated using a pessimistic scenario corresponding to noise levels of $5 \%$; better precisions can be achieved with better signal to noise ratio. 
We conclude that even at modest pressure variations, the assumption of uniform pressure in traditional algorithms can result in unacceptable uncertainty in traditional algorithms.

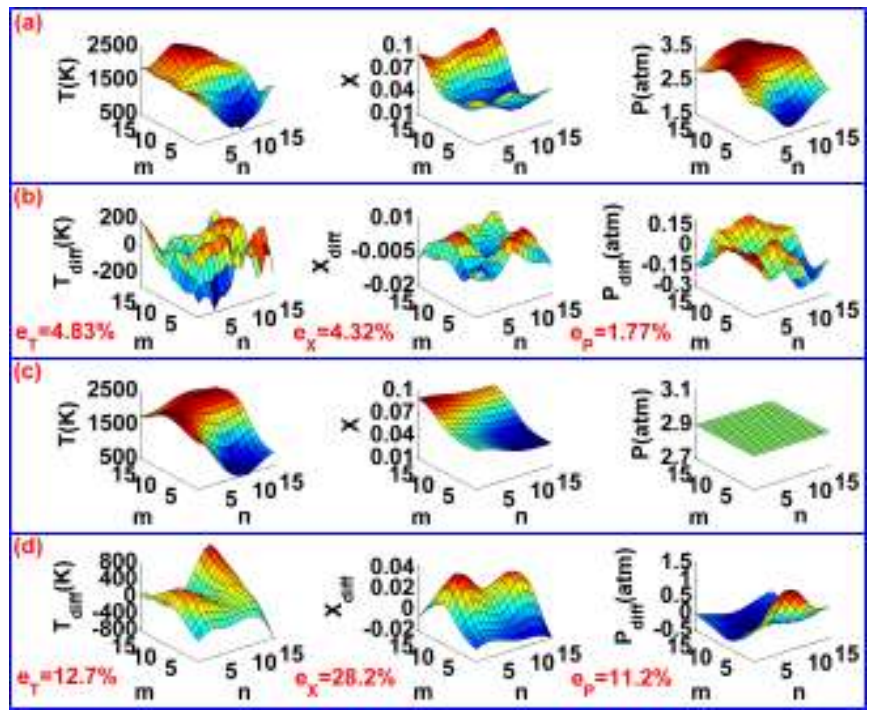

Fig. 4. (Color online) Counterpart of Fig. 3 at high pressure conditions.

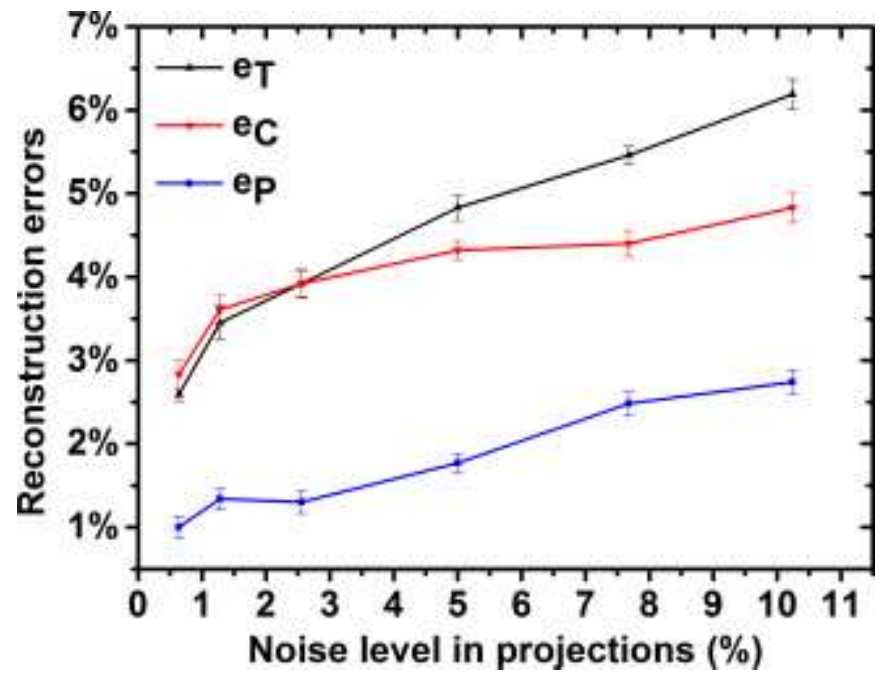

Fig. 5. (Color online) Reconstruction errors as a function of noise levels in projections.

Figure 5 shows the reconstruction errors as a function of noise levels. Shown are reconstruction errors for temperature (black squares), concentration (blue upward triangles), and pressure (red downward triangles) respectively. Error bars represent the standard deviation of 30 cases using projections with the same noise levels. As can be seen, the reconstruction errors increase almost linearly with noise levels facilitating the prediction of errors for a given measurement uncertainty.

To summarize, we demonstrate and validate numerically a new optical tomography technique capable of simultaneously retrieving distributions of temperature, concentration, and pressure. The technique makes use of multispectral absorption spectroscopy. Proof-of-concept simulations were performed on representative flame phantoms and confirmed the validity and robustness of the method. We expected this technique to offer information in quickly fluctuating flows in which nonuniform pressure fields prevail.

This work is supported by the Seventh Framework Program (Grant Agreement Number: PIIF-GA-2012330840) of the European Union and was performed using the Darwin Supercomputer of the University of Cambridge High Performance Computing Service. We would like to acknowledge Dan Holland for useful discussions.

${ }^{1}$ M. G. Allen, Meas. Sci. Technol. 9, 545-562 (1998).

${ }^{2}$ E. J. Beiting, Appl. Opt. 31, 1328-1343 (1992).

${ }^{3}$ C. Belotti, F. Cuccoli, L. Facheris, and O. Vaselli, IEEE

Trans. Geosci. Remote Sensing 41, 2629-2637 (2003).

${ }^{4}$ S. J. Carey, H. McCann, F. P. Hindle, K. B. Ozanyan, D. E. Winterbone, and E. Clough, Chem. Eng. J. 77, 111-118 (2000).

5J. Chen, D. Hou, T. Zhang, and Z. Zhou, Flow Meas. Instrum. 16, 321-325 (2005).

${ }^{6}$ F. Cuccoli, L. Facheris, S. Tanelli, and D. Giuli, IEEE Trans. Geosci. Remote Sensing 38, 1922-1935 (2000).

7M. G. Twynstra and K. J. Daun, Appl. Opt. 51, 70597068 (2012).

${ }^{8}$ W. Cai, D. J. Ewing, and L. Ma, Comput. Phys. Commun. 179, 250-255 (2008).

${ }^{9}$ L. Ma, W. Cai, A. W. Caswell, T. Kraetschmer, S. T. Sanders, S. Roy, and J. R. Gord, Opt. Express 17, 86028613 (2009).

${ }^{10}$ L. Ma, X. Li, S. T. Sanders, A. W. Caswell, S. Roy, D. H. Plemmons, and J. R. Gord, Opt. Express 21, 1152-1162 (2013).

${ }^{11}$ W. Cai, D. J. Ewing, and L. Ma, Applied Mathematics and Computation 217, 5754-5767 (2011).

12J. Hult, R. S. Watt, and C. F. Kaminski, Opt. Express 15, 11385-11395 (2007).

${ }^{13}$ C. Kaminski, R. Watt, A. Elder, J. Frank, and J. Hult, Applied Physics B 92, 367-378 (2008).

14J. Langridge, T. Laurila, R. Watt, R. Jones, C. Kaminski, and J. Hult, Opt. Express 16, 10178-10188 (2008).

15G. P. Agrawal, Nonlinear fiber optics (Springer, 2000).

${ }^{16 R}$. Watt, C. Kaminski, and J. Hult, "High bandwidth $\mathrm{H}_{2} \mathrm{O}$ absorption spectroscopy in a flame using a dispersed supercontinuum source," in Conference on Lasers and Electro-Optics, San Jose, CA, 2008),

${ }^{17}$ R. S. Watt, T. Laurila, C. F. Kaminski, and J. Hult, Applied spectroscopy 63, 1389-1395 (2009).

${ }_{18}^{18}$ T. Laurila, I. Burns, J. Hult, J. Miller, and C. Kaminski, Applied Physics B 102, 271-278 (2011).

19L. Rothman, I. Gordon, R. Barber, H. Dothe, R. Gamache, A. Goldman, V. Perevalov, S. Tashkun, and J. Tennyson, Journal of Quantitative Spectroscopy and Radiative Transfer 111, 2139-2150 (2010).

${ }^{20}$ L. S. Rothman, I. E. Gordon, A. Barbe, D. C. Benner, P. F. Bernath, M. Birk, V. Boudon, L. R. Brown, A. 
Campargue, and J.-P. Champion, Journal of Quantitative Spectroscopy and Radiative Transfer 110, 533-572 (2009).

${ }^{21} \mathrm{H}$. Li, "Near-infrared diode laser absorption spectroscopy with applications to reactive systems and combustion control," (Stanford University, 2007).

${ }^{22}$ K. L. Letchworth and D. C. Benner, Journal of Quantitative Spectroscopy and Radiative Transfer 107, 173-192 (2007).

${ }^{23}$ S. Tedder, S. O'Byrne, P. Danehy, and A. Cutler, AIAA Paper 616, 10-13 (2005). 
(a) Region of interest

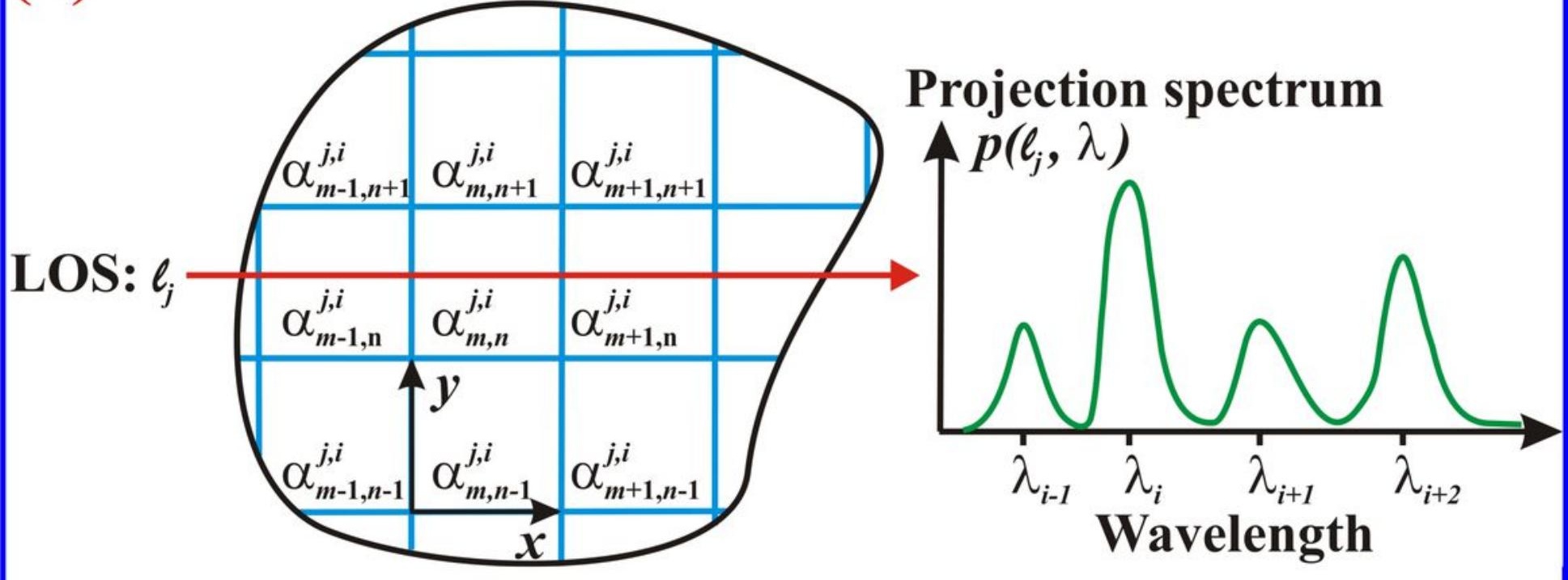

(b)

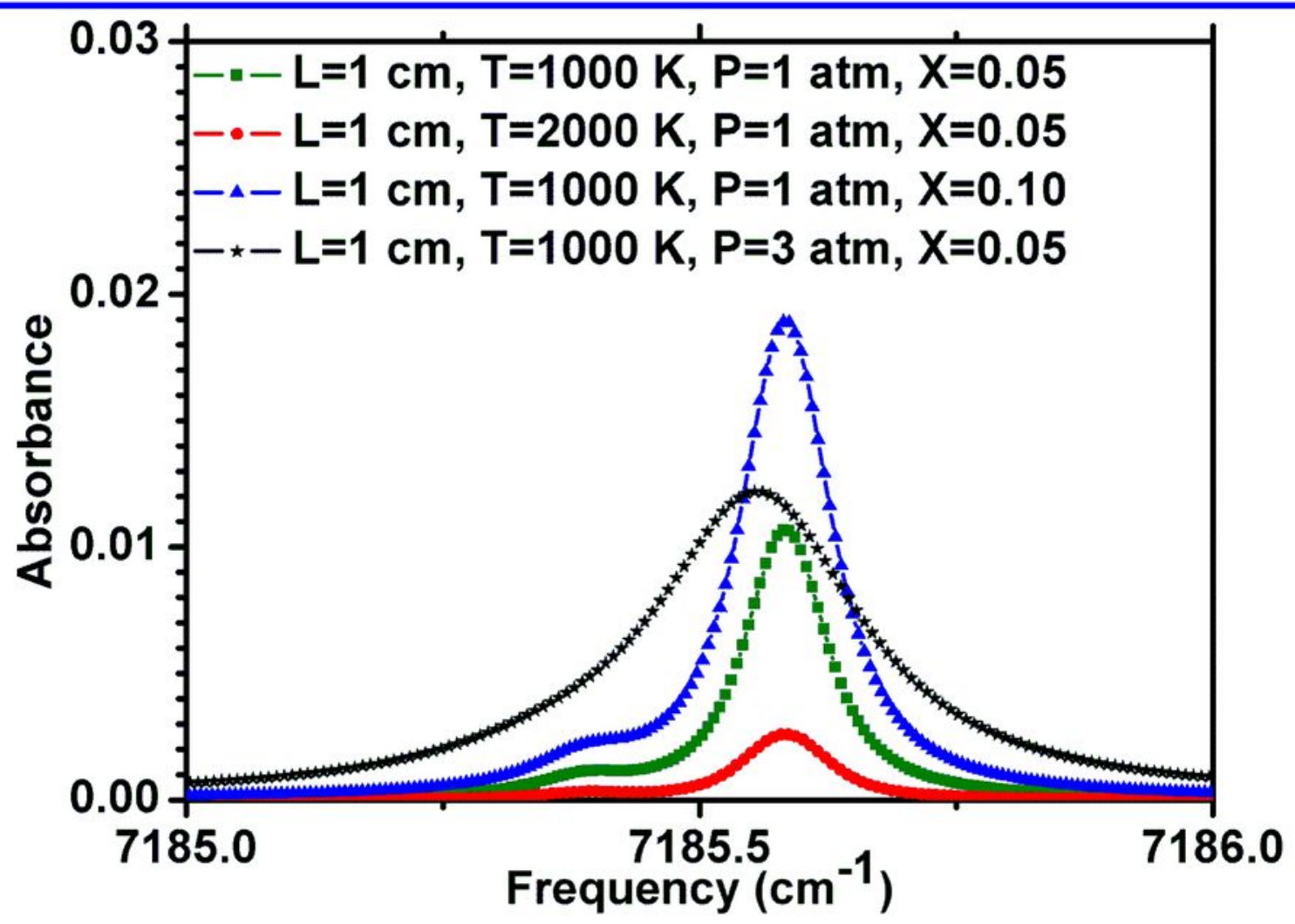

(c)

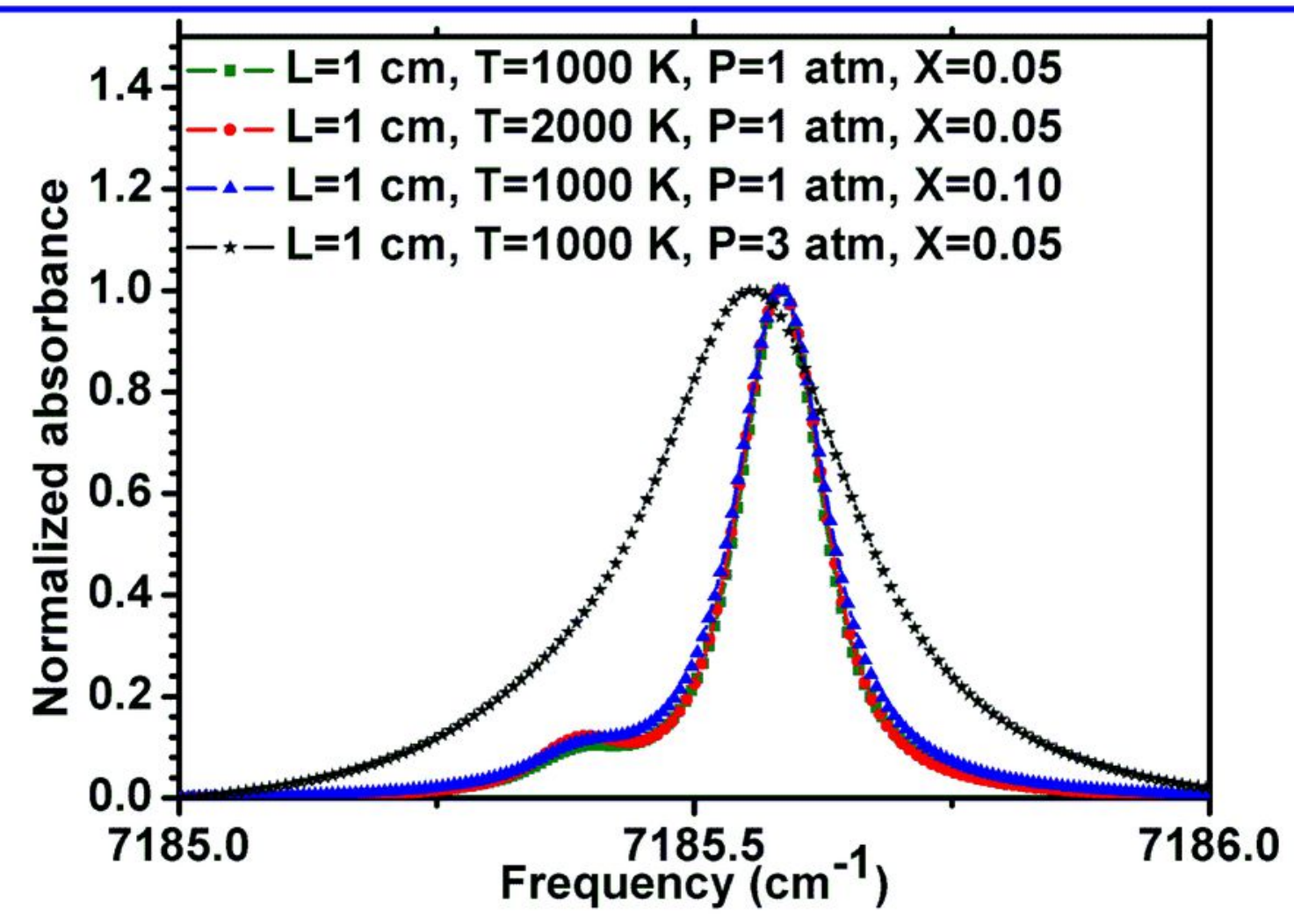


(a)

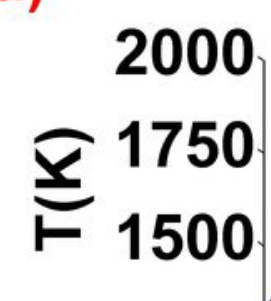

15

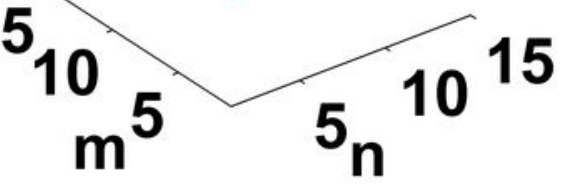

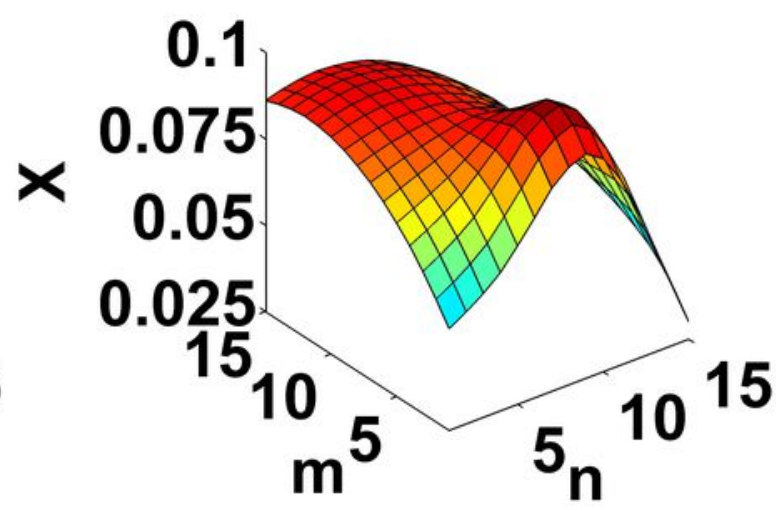

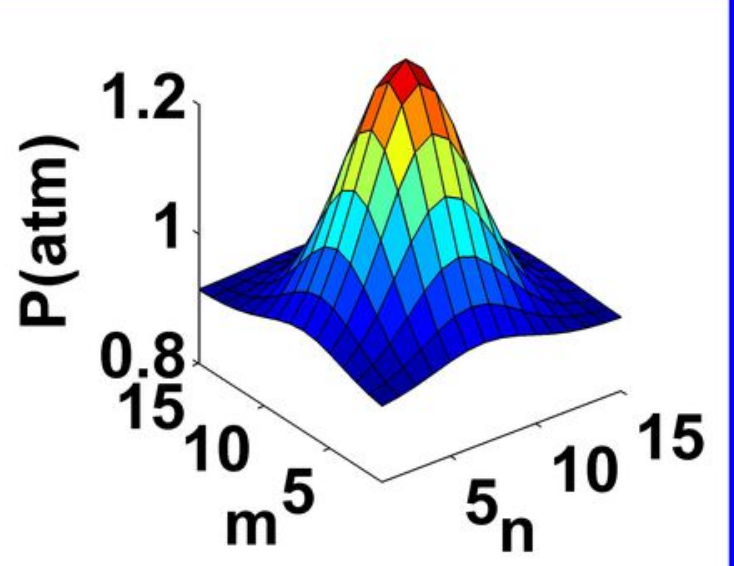

(b)
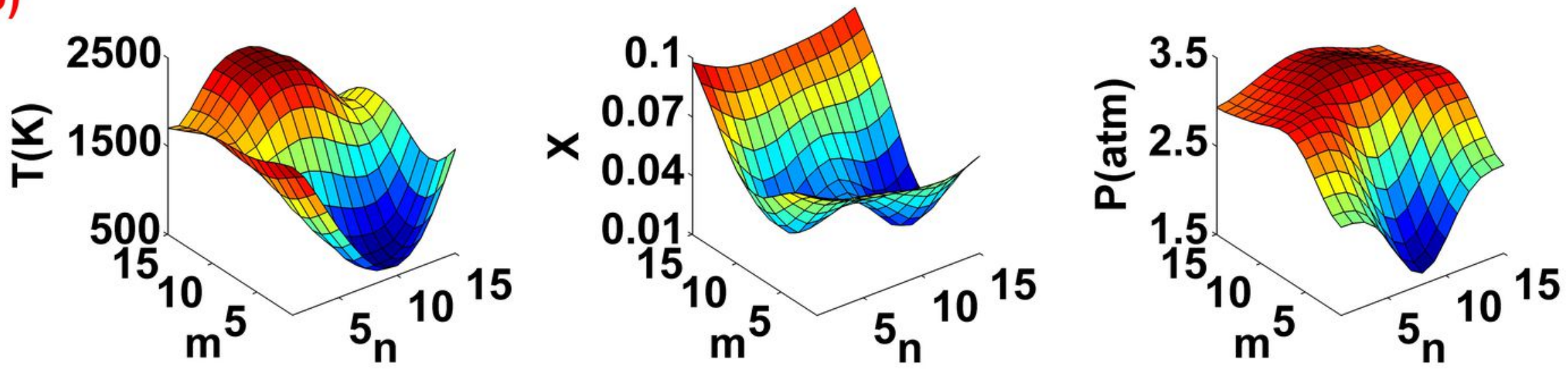

$\mathrm{m}$ 
(a)

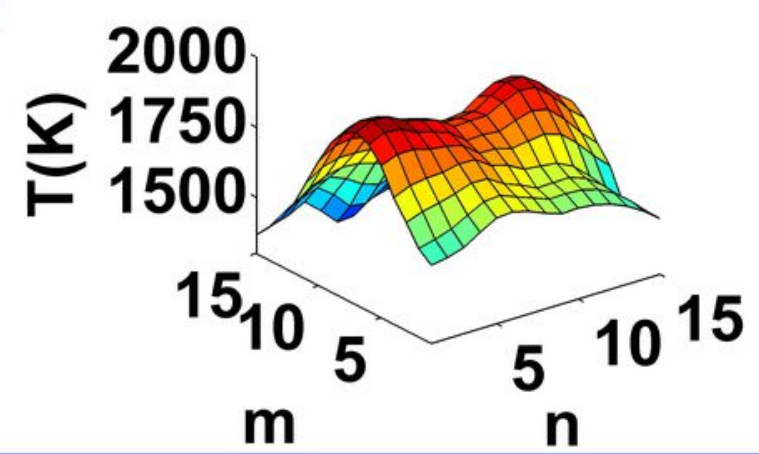

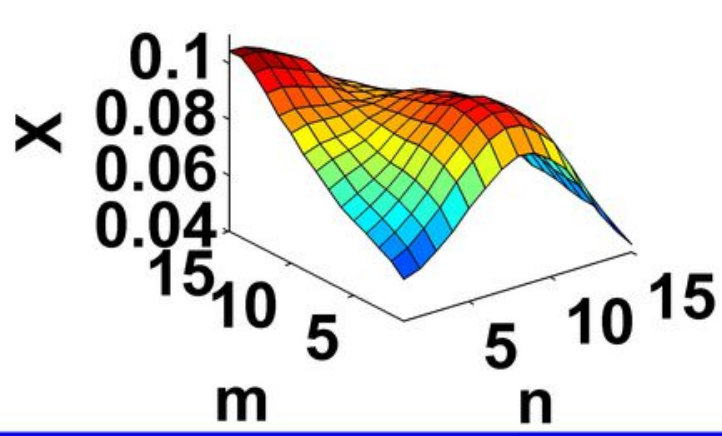

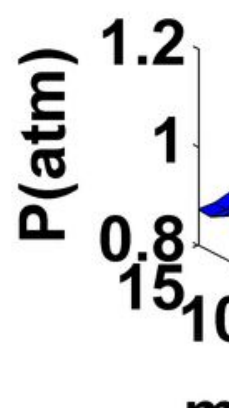
m (b)

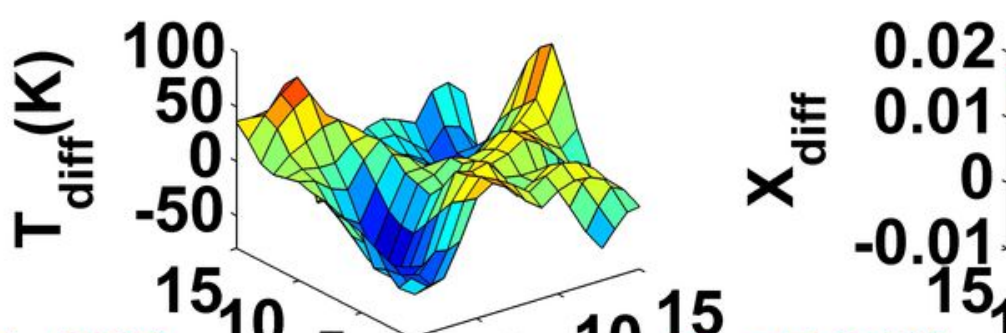

$e_{T}=1.49 \%$ $5 \quad 5 \quad 10 e_{x}^{15}=5.84 \%$

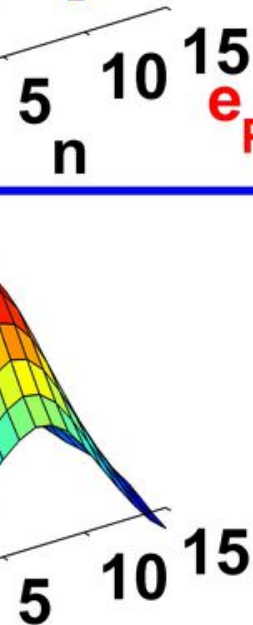

5

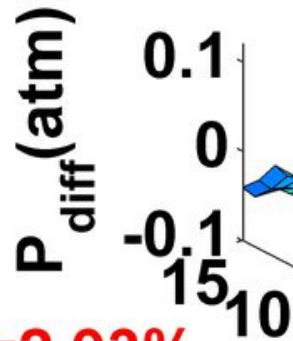

(c)

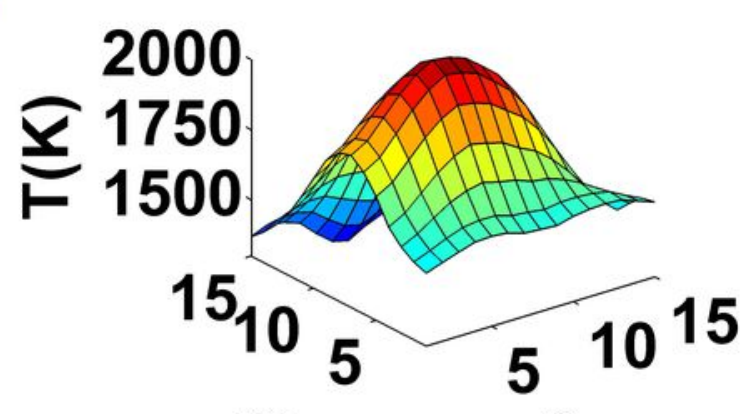

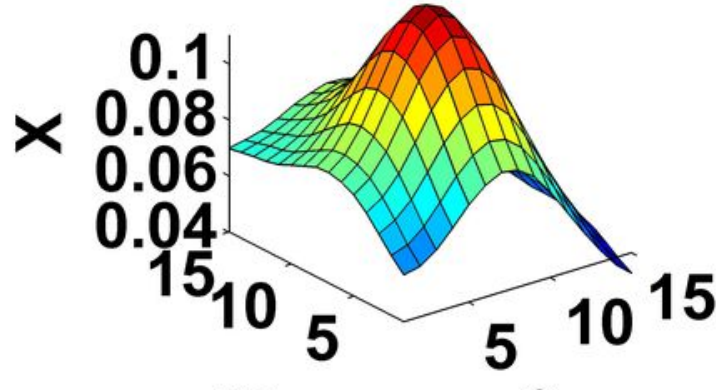

m $\begin{array}{lr}\text { E } & 1.2 \\ \text { 한 } & 1 \\ \text { ㄴ } & 0.8 \\ & 15\end{array}$ m 15 n

(d)

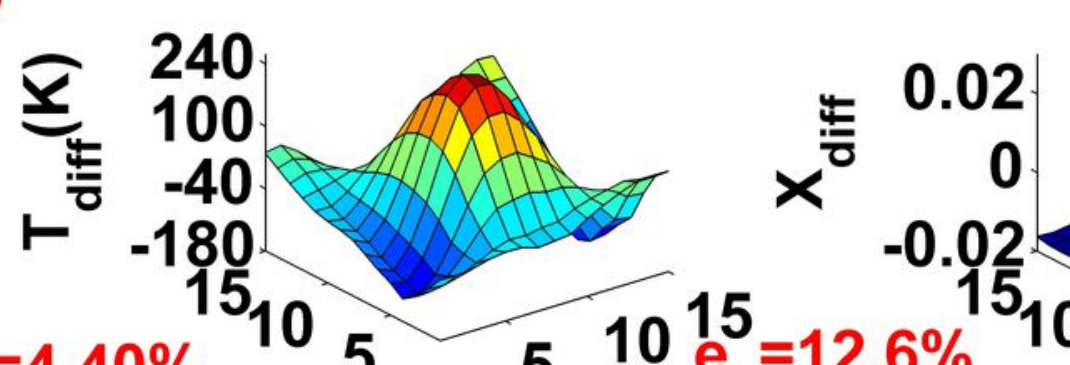


(a)
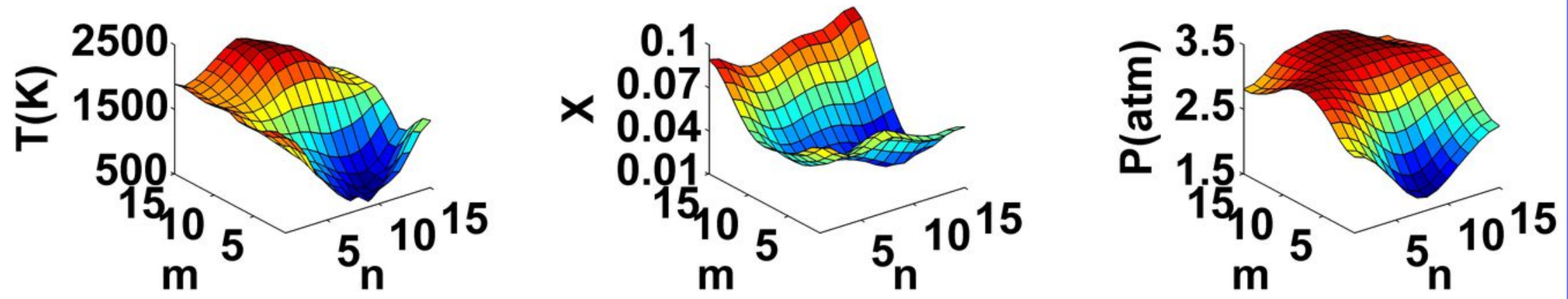

(b)

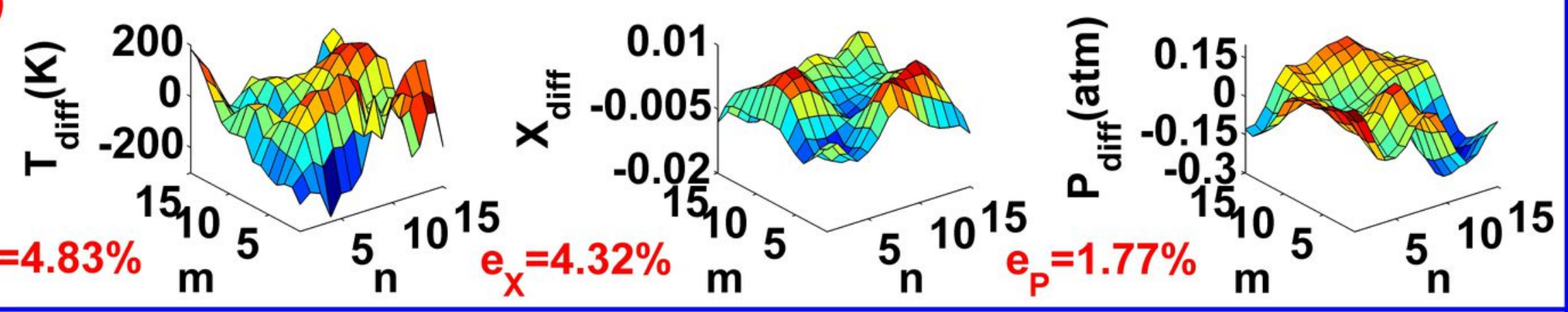

(c)
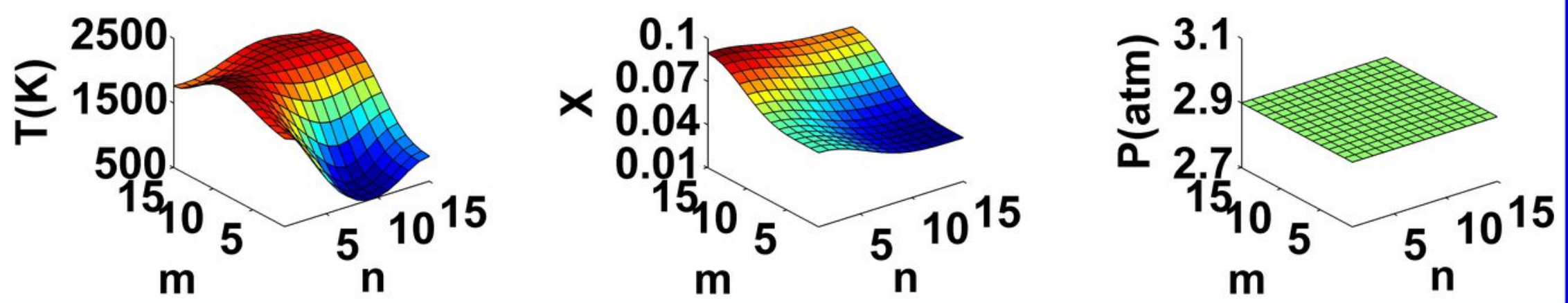

(d)



$e_{T}=12.7 \%$

m

n

$e_{x}=28.2 \%$

m

$5 \quad{ }_{n} 10^{15} e_{p}=11.2 \%$

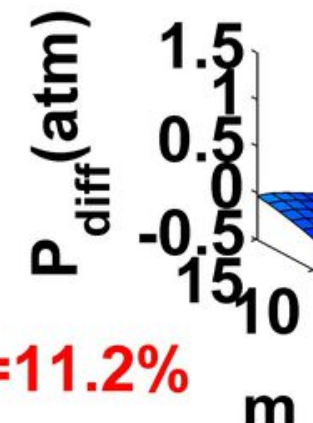

5

m 


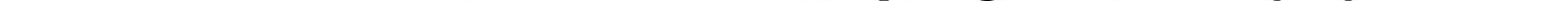

